\title{
A minimal model of peptide binding predicts ensemble properties of serum antibodies
}

\author{
Victor Greiff ${ }^{1,6}$, Henning Redestig ${ }^{1,2,5,6}$, Juliane Lück ${ }^{1}$, Nicole Bruni ${ }^{1,3}$, Atijeh Valai ${ }^{1}$, Susanne Hartmann ${ }^{4}$, \\ Sebastian Rausch ${ }^{4}$, Johannes Schuchhardt ${ }^{5}$ and Michal Or-Guil ${ }^{*}$
}

Background: The importance of peptide microarrays as a tool for serological diagnostics has strongly increased over the last decade. However, interpretation of the binding signals is still hampered by our limited understanding of the technology. This is in particular true for arrays probed with antibody mixtures of unknown complexity, such as sera. To gain insight into how signals depend on peptide amino acid sequences, we probed random-sequence peptide microarrays with sera of healthy and infected mice. We analyzed the resulting antibody binding profiles with regression methods and formulated a minimal model to explain our findings.

Results: Multivariate regression analysis relating peptide sequence to measured signals led to the definition of amino acid-associated weights. Although these weights do not contain information on amino acid position, they predict up to $40-50 \%$ of the binding profiles' variation. Mathematical modeling shows that this position-

independent ansatz is only adequate for highly diverse random antibody mixtures which are not dominated by a few antibodies. Experimental results suggest that sera from healthy individuals correspond to that case, in contrast to sera of infected ones.

Conclusions: Our results indicate that position-independent amino acid-associated weights predict linear epitope binding of antibody mixtures only if the mixture is random, highly diverse, and contains no dominant antibodies. The discovered ensemble property is an important step towards an understanding of peptide-array serum-antibody binding profiles. It has implications for both serological diagnostics and B cell epitope mapping.

\section{Background}

The functional antibody repertoire (FABR), the set of all antibodies produced by plasma cells at any one time, determines the immune system's perception of the antigen universe. The FABR is shaped throughout the life of an individual by various stages and selection events during B cell development that take place in the fetal liver, in the bone marrow and in secondary lymphatic organs. As the FABR is subject to constant change due to continuous antigen encounter and establishment of immunological memory [1], it encompasses a variety of specificities and affinities for a wide range of antigens [2]. The FABR's investigation thus provides the possibility to gather information about both past and on-going

\footnotetext{
* Correspondence: m.orguil@biologie.hu-berlin.de

'Systems Immunology Lab, Department of Biology, Humboldt University Berlin, and Research Center ImmunoSciences, Charité University Medicine Berlin, Berlin, Germany

Full list of author information is available at the end of the article
}

immune responses, and ultimately about the immune state of the body [3].

Since the FABR is highly diverse and the production of antibodies is a hallmark of many infectious and autoimmune diseases, high-throughput immunoblot and microarray technologies have been used intensively for large-scale profiling of serum antibody binding [4-9]. Antibody profiling data is widely used for serological diagnostics by exploiting the fact that sera of control and diseased individuals may differ substantially in their FABRs [7,8,10-12]. Currently, serum-antibody profiling is usually performed by incubating a serum sample with a peptide or protein microarray. Afterwards, the reactivity of antibodies is estimated by measuring the fluorescence from a fluorochrome-coupled secondary antibody that binds to the constant region of the subset of serum antibodies studied [13,14].

The importance of peptide microarrays as a tool for serological diagnostics has strongly increased over the
Ciomed Central

() 2012 Greiff et al; licensee BioMed Central Ltd. This is an Open Access article distributed under the terms of the Creative Commons Attribution License (http://creativecommons.org/licenses/by/2.0), which permits unrestricted use, distribution, and reproduction in any medium, provided the original work is properly cited. 
last decade. However, interpretation of the binding signals is still hampered by our limited understanding of the technology [15]. This is in particular true for arrays probed with antibody mixtures of unknown complexity, such as sera. To gain insight into how signals depend on peptide amino acid sequences, we probed randomsequence peptide microarrays with sera of healthy and infected mice.

For prediction of antibody binding profiles, we use a multivariate regression model based exclusively on the peptide library's amino acid composition without taking into account amino acid positional information. This approach is related to methods of linear B cell epitope prediction which rely on propensity scales for epitope prediction [16-19]. Our method contrasts, however, with previously reported quantitative structure-activity relationship (QSAR) modeling which, in conjunction with physico-chemical properties, relates amino acid positions and amino acid compositions of peptides and monoclonal antibodies to various response variables [20-22]. We propose to examine, in vitro and in silico, the extent to which the validity of our approach depends on the composition of antibody mixtures.

The regression model led to the definition of amino acid-associated weights (AAWS) as predictors of antibody-peptide reactivity. We found that the positionindependent peptide amino acid composition accounts for up to $40-50 \%$ in variation of antibody-peptide binding for healthy mice.

We demonstrate with a mathematical model the ensemble properties of highly diverse, random antibody mixtures in which no antibody dominates. We call these mixtures "unbiased" and show that the properties of unbiased mixtures are the foundation to a high predictive performance of AAWS. We hypothesize that serum antibodies of healthy individuals resemble an unbiased mixture, while during an acute immune response, specific antibodies dominate antibody-peptide binding thus lowering predictive performance. Based on in silico and in vitro evidence, our work thus suggests that the faithfulness of antibody-peptide binding prediction with propensity scales [16-19] decreases with increasing antibody dominance in a mixture.

\section{Results}

In order to investigate the binding of antibody mixtures to large random-sequence peptide libraries, we asked two main questions: i) what is the impact of the peptides' amino acid composition on the binding to serum antibodies, ii) and how does the serum-antibody composition influence binding prediction?

\section{Experimental setup}

To study the impact of amino acid composition of random-sequence peptide libraries on measured signal intensity, serum samples from $15 \mathrm{BALB} / \mathrm{c}$ mice bred under specific pathogen-free (SPF) conditions were collected. These mice were infected with HB (Additional file 1, Figure S1). Further serum samples were collected at $10 \mathrm{dpi}$ (days post infection; 15 samples), at $14 \mathrm{dpi}$ (13 samples) and at $18 \mathrm{dpi}$ (15 samples) totaling 58 serum samples. Microarrays of $n_{\text {Pep }}=255$ random-sequence peptide probes (hereafter referred to as standard library) were incubated with the serum samples. The peptide arrays used have been shown to be suitable for serological diagnostics by Bongartz et al. [10]. Each probe consisted of $l=14$ out of 20 proteinogenic amino acids. IgM and IgG antibody binding was simultaneously detected by means of isotype specific fluorochromelabeled polyclonal secondary antibodies. In addition to serum samples, the peptide library was incubated separately with 13 different human monoclonal IgG antibodies.

The fluorescence signal intensities were read, log-transformed and corrected for the signal from the polyclonal secondary antibody binding directly to the peptide probes. Subsequently, the signal intensities were meancentered and scaled to unit variance, which resulted in a normalized vector $\vec{s}$ for each IgM and IgG serum sample and for each of the 13 monoclonal antibodies. We use the terms signal intensity or antibody binding profile interchangeably to denote $\vec{s}$. Each signal intensity vector $\vec{s}$ has as many components as there are peptides in the standard random peptide libary. For brevity, our analysis focuses on the IgM data. The IgG data can be found in the Supporting Information (Additional file 2, Figure S2, Additional file 3, Figure S3, and Additional file 4, Figure S4). More details on the experimental setup and normalization procedures can be found in Methods.

\section{A regression model based exclusively on peptide amino acid composition predicts antibody binding profiles}

We built a linear statistical model to relate the amino acid composition of our peptide library to measured signal intensities

$$
\vec{s}=\mathbf{X} \vec{w}+\vec{\varepsilon},
$$

where $\vec{s}(255 \times 1)$ is the signal intensity vector and $\mathbf{X}$ the amino acid composition matrix (AACM) of the peptide library. The $\mathbf{X}$ matrix is formed by counting the occurrences of each of the 20 amino acids in each peptide which results in a matrix with 20 columns and 255 rows. Importantly, $\mathbf{X}$ does not contain information about the position of an amino acid in a given peptide sequence.

The AAWS vector $\vec{w}(20 \times 1)$ indicates the contribution of every amino acid to the measured signal intensity. Furthermore, the residual of the regression model, 
$\vec{s}$, captures the part of $\vec{s}$ which cannot be explained by $\mathbf{X}$ alone. AAWS and residuals were estimated by partial least squares regression (PLS) (see Methods for details on the data analysis).

Once the vector $\vec{w}$ has been estimated, we use the regression model to predict measured signal intensities given the peptides' amino acid composition. Figure 1 illustrates that the predicted signal intensities $\hat{\vec{s}}=\mathbf{X} \vec{w}$ are in good agreement with signal intensities $\vec{s}$ measured for the serum of one healthy BALB/c mouse. In order to evaluate the performance of the regression model, we focus on the predictive performance, $Q^{2}$, which was determined by 10 -fold cross-validation (Methods). The predictive performance equals 1 for perfect predictions and is close to zero for poor predictions.

All $58 \mathrm{BALB} / \mathrm{c}$ serum samples resulted in a median predictive performance of 0.39 (Figure 2).

\section{A minimal model of antibody-peptide binding}

We hypothesize that the high predictive performance of our regression model is due to properties of an antibody ensemble. We test this hypothesis with the help of a model that simulates binding between peptides and antibodies. In this model, the binding affinity of simulated monoclonal antibodies depends non-linearly on amino acid positions in the peptide sequences (Equations 2 and 4 ). The model we propose is similar to bit string models [23-26] in that it uses vectors as simple representations of peptides and antibodies. The peptide string is represented by unique real numbers taken from a vector of assigned AAWS, denoted $\vec{h}$, the twenty components of which were drawn from a uniform distribution on the closed interval $0[1]$. A peptide $\overrightarrow{p^{i}}$ of $l$ amino acids is thus represented by a vector of $l$ numbers drawn from $\vec{h}$.

An antibody binding site is represented by a vector $\overrightarrow{a^{k}}$ of length $l$. The binding strength of each position is given by a number between -1 and 1 that is drawn randomly from a uniform distribution and is scaled such that $\overrightarrow{\left(a^{k}\right)^{\mathrm{T}}} \overrightarrow{a^{k}}=1$. The binding association between peptide $\overrightarrow{p^{i}}$ and antibody $\overrightarrow{a^{k}}$ is computed as the dot product of the two vectors, $y_{i, k}=\left(\overrightarrow{a^{k}}\right)^{\mathrm{T}} \overrightarrow{p^{i}}$. Thus, the binding association $y_{i, k}$ depends explicitly on an amino acid's position in a given peptide sequence.

An expression for the simulated signal intensity, based on the law of mass action, can be obtained from classical Langmuir adsorption theory [27]:

$$
S_{i}=\frac{\sum_{k=1}^{n_{\mathrm{Ab}}}[\mathrm{Ab}]_{k} K_{i, k}}{1+\sum_{k=1}^{n_{\mathrm{Ab}}}[\mathrm{Ab}]_{k} K_{i, k}}
$$

where $[\mathrm{Ab}]_{k}$ is the concentration of antibody $k$ with $\sum_{k=1}^{n_{\mathrm{Ab}}}[\mathrm{Ab}]_{k}=1$. The thermodynamic equilibrium

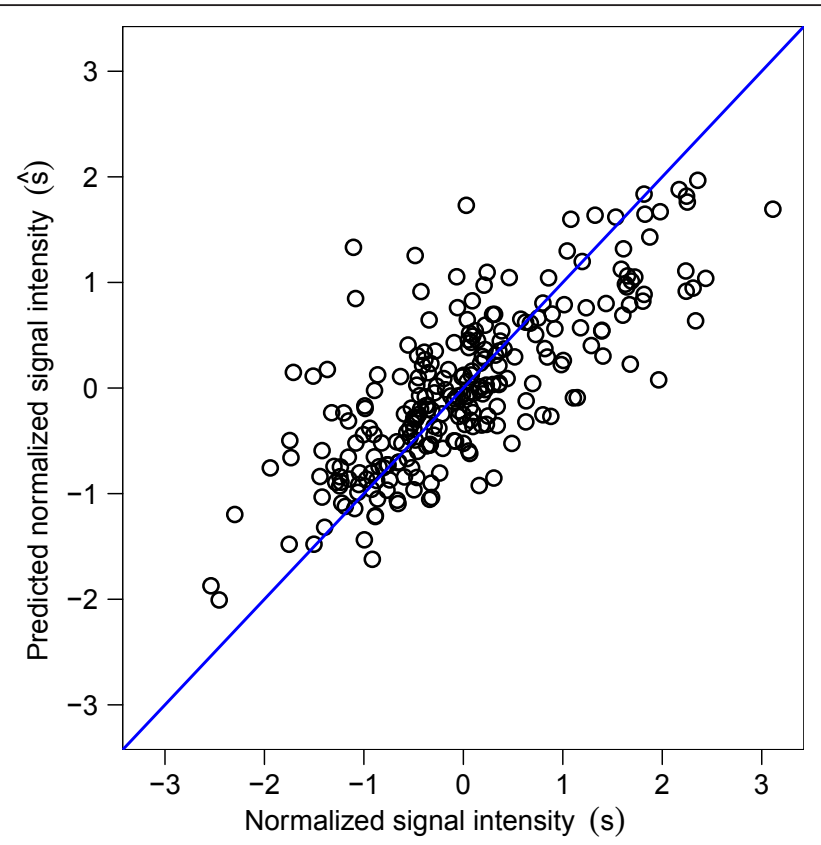

Figure 1 Predicted signal intensity $(\hat{\vec{s}})$ against measured signal $\vec{s}$ of a healthy BALB/c mouse serum sample. The prediction depends exclusively on the amino acid composition of the peptide sequences and is based on the regression model (Equation 1). Predictive performance: $Q^{2}=0.5$. Signal intensities were measured with the standard peptide library of 255 14-mers. 


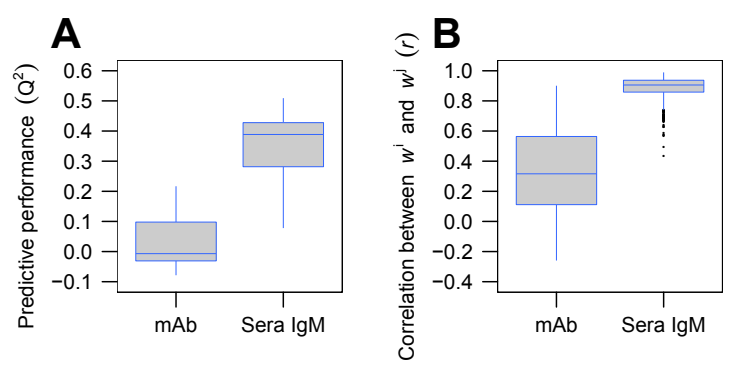

Figure 2 Predictive performance and pairwise correlation of AAWS are higher for serum IgM than for monoclonal antibodies. (A) Predictive performance values were calculated for monoclonal (mAb) and serum IgM antibody (Sera IgM) binding profiles. (B) Shown is the pairwise correlation ( $r$ ) of the corresponding AAWS $\vec{w}^{j}$. In both (A) and (B), mAb signifies antibody binding profiles from 13 monoclonal antibodies and Sera IgM binding profiles from 58 BALB/c mouse serum samples. Differences between monoclonal and serum IgM antibodies in predictive performance $\left(Q^{2}\right)$ and pairwise correlation $(r)$ of AAWS are significant $(p<0.001)$. Antibody binding profiles were measured with the standard peptide library of 255 14-mers. Corresponding AAWS $\left(\vec{w}^{j}\right)$ were determined using Equation 1.

association constant for antibody $k$ binding peptide $i$ is defined $\quad$ as $\quad K_{i, k}=\exp \left(-\frac{\Delta_{r} G^{\mathrm{o}}}{R T}\right) \quad$ with $\Delta_{r} G=\exp \left(\frac{\beta_{0}+\beta_{1} \gamma_{i, k}}{R T}\right)$. Logarithmizing the results of Equation 2, and centering them to zero and unit variance, we obtained a vector of normalized simulated signal intensities $\vec{s}_{\text {sim }}$. A more detailed description of the mathematical model can be found in Methods.

Simulations show that the prediction of antibody binding profiles based exclusively on peptide amino acid composition improves with increasing antibody diversity We first simulated signal intensities for $n_{\mathrm{Ab}}=150$ binding to a simulated peptide library of 255 14-mers. The peptide library used in the simulation determines the amino acid composition matrix $\mathbf{X}_{\text {sim }}$. We estimated simulated intensities $\vec{s}_{\text {sim }}$ (Figure $3 \mathrm{~A}$ ) and respective weights $\vec{w}_{\text {sim }}$ (Figure $3 \mathrm{~B}$ ) using the linear regression model $\hat{\vec{s}}_{\text {sim }}=\mathbf{X}_{\text {sim }} w_{\text {sim }}$. Prediction of simulated signal intensities yielded a predictive performance $\left(Q^{2}\right)$ of 0.40 , and the correlation between $\vec{h}$ and $\vec{w}_{\text {sim }}$ was found to be $r=0.92$ (Figure 3B), which indicates a very good recovery of $\vec{h}$. Recall that signal intensities were simulated in an amino acid position-dependent manner, while the composition-based regression model (Equation 1) relies on the amino acid position-independent matrix $\mathbf{X}_{\text {sim. }}$.

Further, our simulation framework enabled us to show in silico that the predictive performance increases with growing antibody diversity (Figure 4A). The same is true for the pairwise correlation of computed AAWS $\left(\vec{w}_{\text {sim }}^{i}\right)$, which nears perfection $(r=1)$ with increasing antibody diversity (Figure 4B), as does the correlation of AAWS with $\vec{h}$ (Additional file 5, Figure S5). Therefore, when using a position-independent linear statistical model for the prediction of antibody-peptide binding, high antibody diversity is a prerequisite for good predictive performance.

\section{Predictive performance differs for monoclonal and serum-} antibody binding profiles

In order to test our in silico-based prediction that predictive performance depends heavily on antibody diversity when only taking into account the peptide library's amino acid composition, we compared the predictive performance of the $58 \mathrm{BALB} / \mathrm{c}$ mouse serum samples (antibody diversity $n_{\mathrm{Ab}}>>1$ ) with that of the 13 human monoclonal IgG antibodies (antibody diversity $n_{\mathrm{Ab}}=1$ ). We found both a significantly higher predictive performance (Figure 2A, p < 0.001) and significantly higher pairwise correlations between AAWS for serum antibodies (Figure $2 \mathrm{~B}, \mathrm{p}<0.001$ ) than for monoclonal antibodies, which confirms the predictions of our mathematical model (Figure 4).

\section{Predictive performance decreases in the course of an HB- infection}

In order to quantify the influence of immune response stage during $\mathrm{HB}$-infection on predictive performance, we divided the mouse serum samples into three groups: healthy, acute phase (10 and $14 \mathrm{dpi}$ ), and early chronic phase (18 dpi) [28]. We found that predictive performance (Figure 5A) and pairwise correlation of AAWS decrease significantly in the course of the immune response (Figure 5B).

In order to compare the experimental results with the mathematical model, we simulated signal intensities for 100 random mixtures of 16000 different antibodies (Figure $6 \mathrm{~A}$ and $6 \mathrm{~B}$, case I) and found that, when multiplicative Gaussian noise is introduced into the simulated signal intensities, both predictive performance and pairwise correlation of AAWS decrease (Figure 6A and 6B, case II). By increasing the concentration of one 

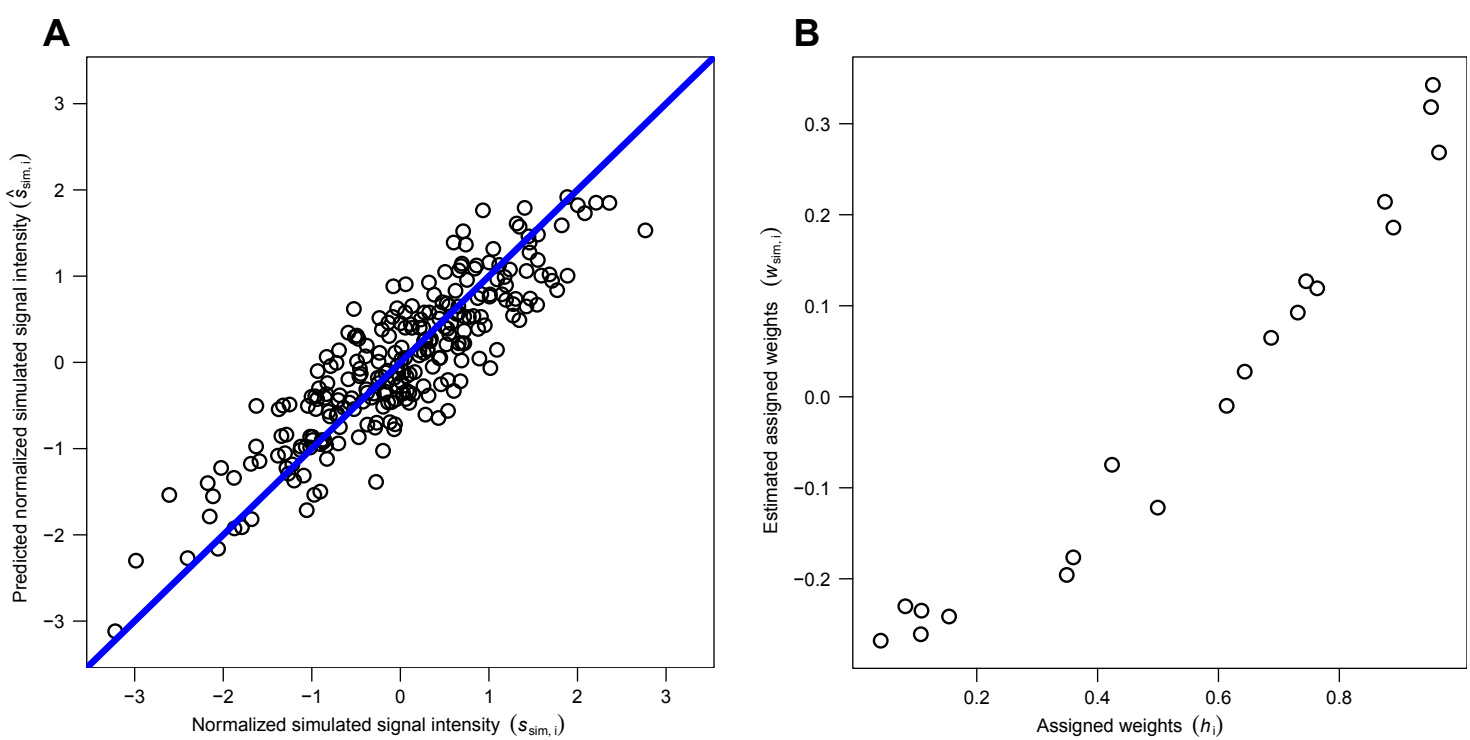

Figure 3 Simulated signal intensities and assigned amino acid-associated weights are recovered by an amino acid composition-based regression model. (A) Simulated signal intensities $\left(\overrightarrow{\boldsymbol{s}}_{\text {sim }}\right)$ obtained by amino acid position-dependent simulation of the binding of 150 antibodies to an array of 255 14-mer peptides were predicted using the regression model given by Equation 1. This regression model takes into account only the amino acid composition of the simulated peptide sequences. Predictive performance: $Q^{2}=0.40$. Simulated signal intensities were computed using Equation 2. (B) Equation 1 was used to estimate $\left(\vec{w}_{\text {sim }}\right)$, which are shown against the assigned AAWS $(\vec{h})$ used to generate the simulated signal intensities $\overrightarrow{\boldsymbol{s}}_{\text {sim }}$. Coefficient of correlation: $r=0.92$.

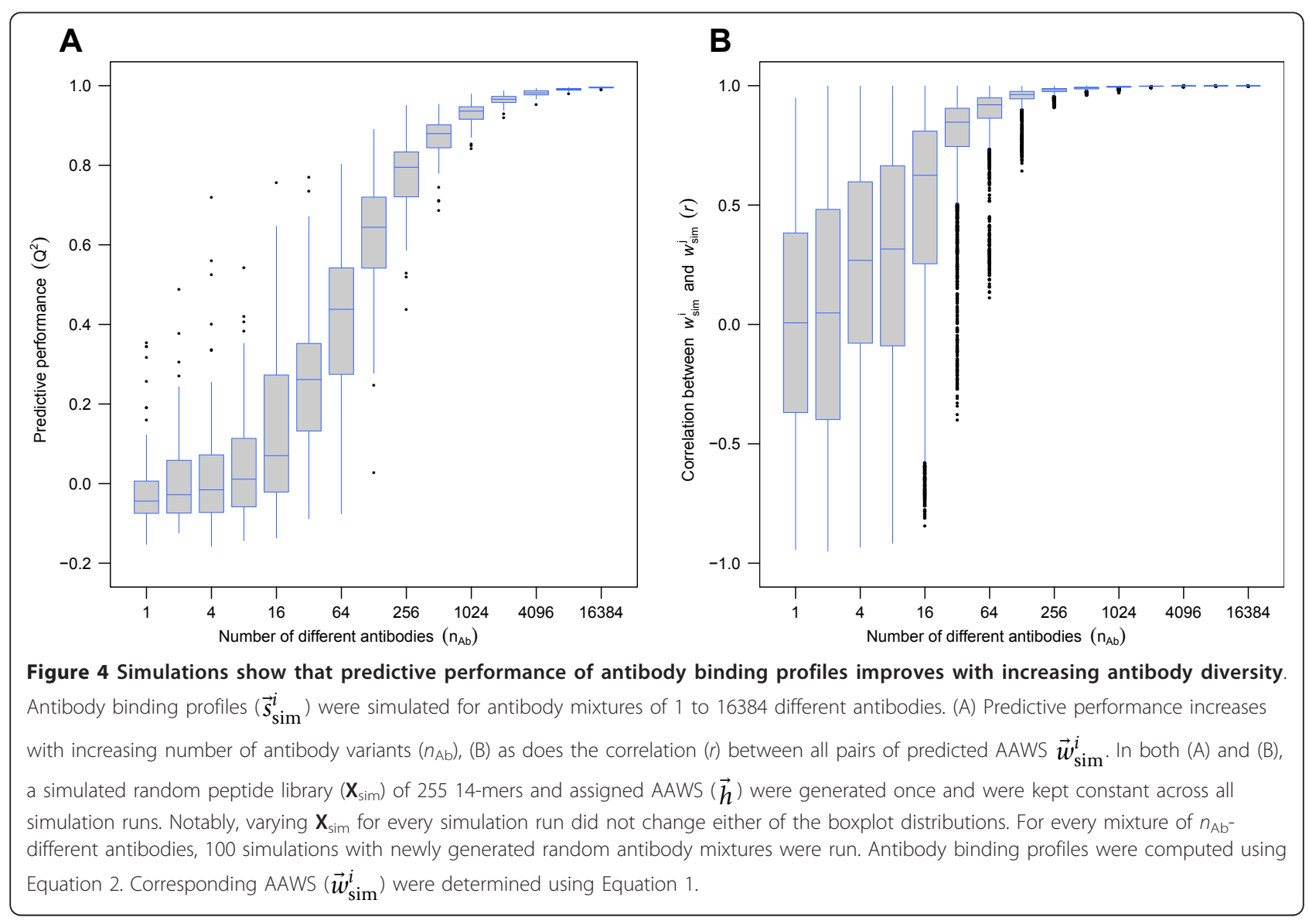



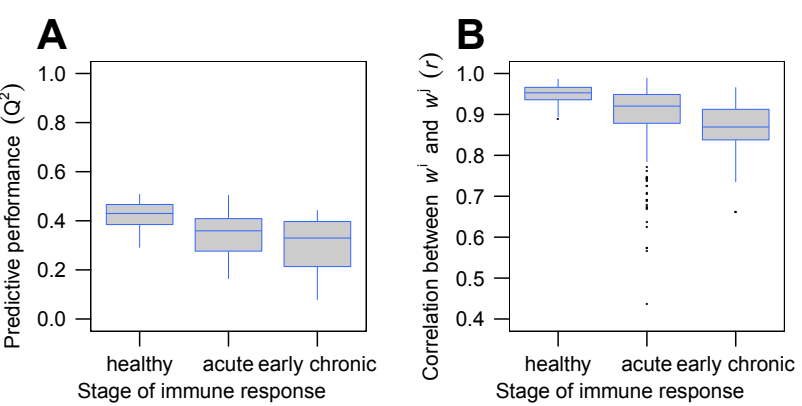

Figure 5 Predictive performance and pairwise correlation of AAWS decrease for serum IgM antibodies in the course of the HBinfection. (A) Predictive performance values $\left(Q^{2}\right)$ were computed from serum IgM antibody binding profiles across three stages of immune response: healthy, acute, early chronic. (B) Shown is the pairwise correlation (r) of the corresponding AAWS $\vec{w}^{j}$. Numbers of BALB/c mouse serum samples: 15 samples from healthy mice; after infection with HB: 15 samples at $10 \mathrm{dpi}$ and 13 samples at 14 dpi (acute phase), and 15 samples at $18 \mathrm{dpi}$ (early chronic) totaling $58 \mathrm{BALB} / \mathrm{c}$ mouse serum samples. Differences in predictive performance $\left(Q^{2}\right)$ between healthy and both acute phase and early chronic phase mice are significant $(p<0.01)$, as are differences in pairwise correlation $(r)$ between all three stages of immune response $(p<0.001)$. Antibody binding profiles were measured with the standard peptide library of 25514 -mers. Corresponding AAWS ( $\vec{w}^{j}$ ) were computed using Equation 1.

monoclonal antibody (the dominant antibody) to a sufficiently high level (Figure 6A and 6B, cases III and IV), predictive performance is decreased.

\section{Stages of murine immune response differ in their amino acid-associated weights}

In order to test whether the AAWS determined for all $58 \mathrm{BALB} / \mathrm{c}$ mouse serum samples were systematically different from one another, we applied principal component analysis to them. Together, the first two principal components yield a strong separation of healthy and diseased mice. Also, acute and early chronic samples separate (Figure 7). Thus, during an immune response against $\mathrm{HB}, \mathrm{AAWS}$ change in a systematic way.

\section{Average amino acid-associated weights of healthy mice correlate with amino acid physico-chemical properties but not with widely used amino acid scales for epitope prediction}

Because of both the good predictive performance and the high pairwise correlation of AAWS of healthy $\mathrm{BALB} / \mathrm{c}$ mice, we considered their average AAWS as representative of healthy $\mathrm{BALB} / \mathrm{c}$ mice (Figure 8 ). The differences between weights in Figure 8 indicate the
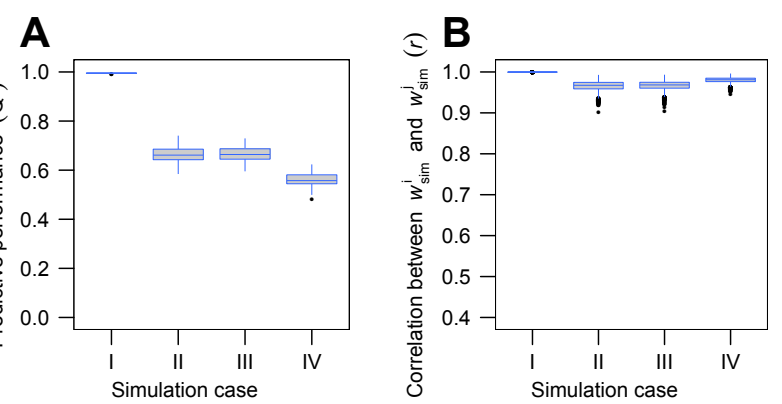

Figure 6 Predictive performance and pairwise correlation of simulated AAWS decrease both with introduction of multiplicative Gaussian noise and antibody dominance. (A) Predictive performance $\left(Q^{2}\right)$ and (B) pairwise correlation of AAWS $(r)$ for different simulated cases. (I) For a given peptide library and given assigned AAWS $(\vec{h})$ we simulated 100 realizations of binding profiles for a mixture of 16000 different antibodies. (II) Same as in I, but Gaussian multiplicative noise was introduced into the simulated signal intensities. (III) Same as in II, but the concentration of a single antibody (dominant antibody) was increased 10-fold. (IV) Same as in II, but concentration of one (dominant) antibody was increased 1000-fold. For both (A) and (B) a simulated peptide library $\left(\mathbf{X}_{\text {sim }}\right)$ and assigned AAWS $(\vec{h})$ were generated once and kept constant across the entire simulation. Simulated antibody binding profiles $\left(\overrightarrow{\boldsymbol{s}}_{\text {sim }}^{i}\right)$ were computed using Equation 2. Corresponding AAWS $\left(\vec{w}_{\text {sim }}^{j}\right)$ were computed using Equation 1. In each of the 100 runs, a newly generated random antibody mixture of $n_{\mathrm{Ab}}=15999$ different antibodies was simulated to which the dominant antibody was added. This antibody was randomly generated once at the beginning of the simulation and was kept constant across all four simulation cases. Gaussian noise term: $\mathcal{N}(\mu=0, \sigma=0.01)$. 


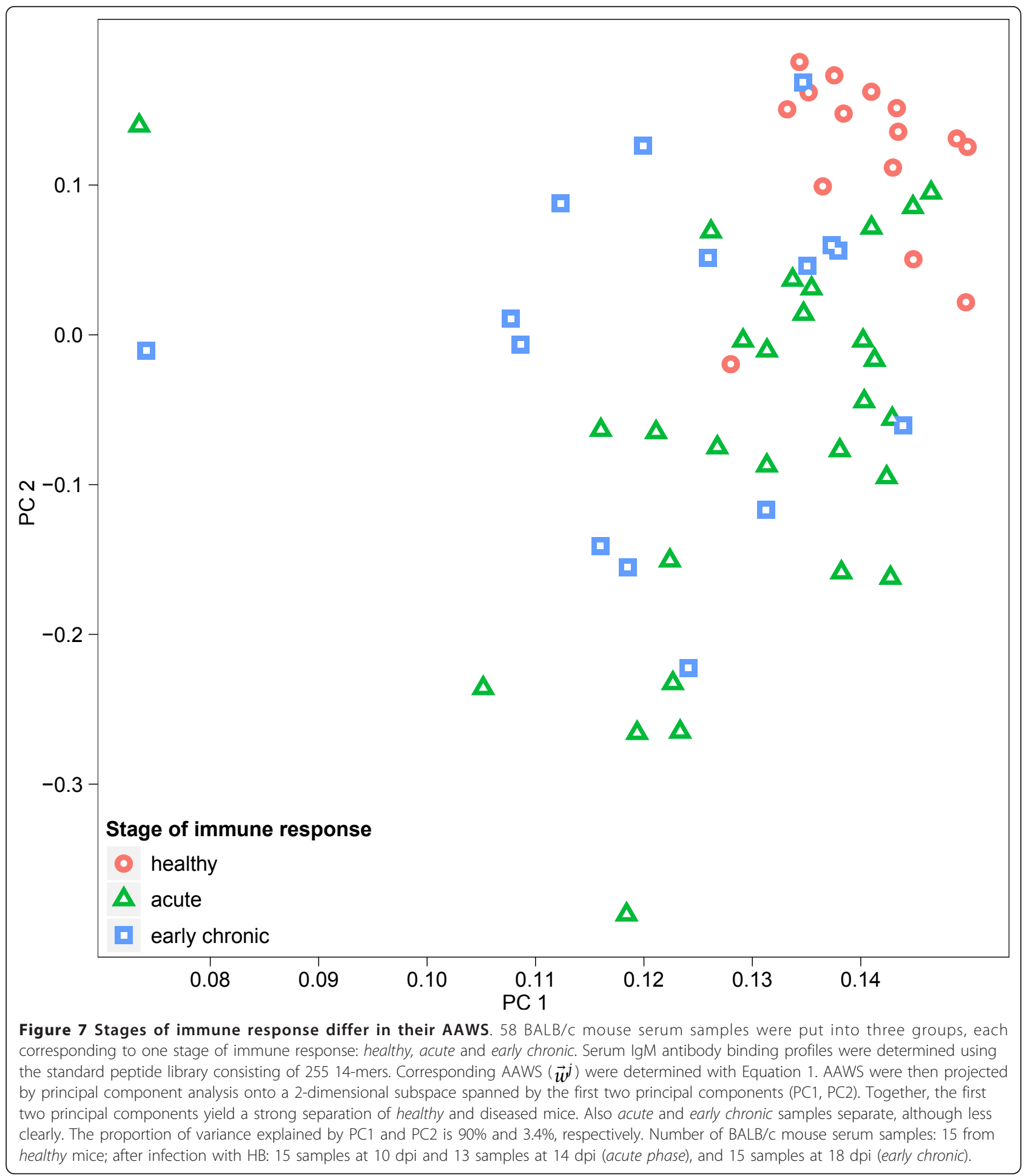

difference in contribution to normalized signal intensity corresponding to an amino acid substitution. Tryptophan, phenylalanine and tyrosine, all of which have aromatic residues, contribute most to the signal intensity.

AAWS represent a priority scale for peptide-antibody binding assigning to every amino acid the importance of contribution to the measured (or simulated) signal intensity. In addition, analogously to QSAR modeling, AAWS can a posteriori be conceived of as a vector representing correlates of the respective amino acids' physico-chemical properties. We therefore correlated the average AAWS (Figure 8) with the $\mathrm{z}$-scale developed 


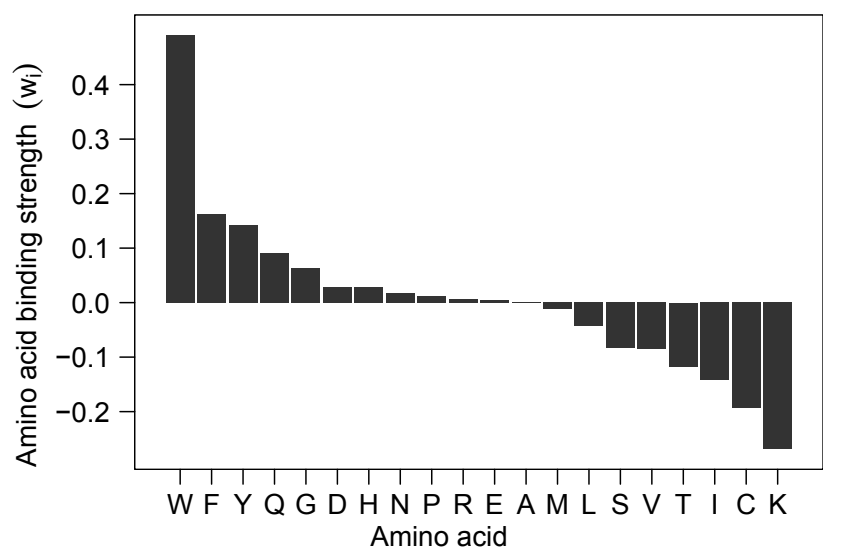

Figure 8 Average recognition of peptides by serum samples of healthy BALB/c mice as given by their AAWS. AAWS of the 15 healthy BALB/C mice (Figure 5A, B; left boxplot) were determined with Equation 1 and averaged. The difference between two weights indicates the contribution to the difference in normalized signal intensity corresponding to an amino acid substitution.

by Sandberg and colleagues [29]. The z-scale aggregates in matrix form 26 physico-chemical amino acid properties for every one of the 20 examined amino acids (Additional file 6, Figure S6). The average AAWS yield an absolute correlation coefficient higher than 0.3 with the following physico-chemical properties: side chain van der Waals volume, alpha-polarizability, absolute electronegativity, number of hydrogen bond donors, total accessible molecular surface area, and indicator of negative charge in side chain.

In order to compare the average AAWS with other published amino acid-scales for epitope prediction, we correlated them with propensity scales published by Parker and colleagues [17] (hydrophilicity), Kolaskar and Tongaonkar [30] (antigenicity), Chou and Fasman [16] (secondary structure) and by Emini and colleagues [18] (accessibility) and found the resemblance with them to be poor (absolute values of correlation coefficients smaller than 0.22 ). Notably, the compared propensity scales also do not highly correlate (range of correlation coefficients: -0.61 to 0.67 ).

\section{Discussion}

Amino acid-associated weights are a compact, information-preserving representation of serum-antibody binding profiles

A minimal linear regression model defines AAWS as predictors that are based solely on the amino acid composition of a given peptide. For serum antibodies of BALB/c mice, AAWS account for up to $50 \%$ of variation in antibody binding profiles, whereas monoclonal antibodies generally show poor predictive performance values. The regression model performs best for healthy mice (median $Q^{2}=0.43$, Figure 5). Furthermore, we find AAWS to be comparable across healthy BALB/c mouse serum samples (Figure 5B). During the immune response against $\mathrm{HB}$, however, predictive performance decreases steadily. Accordingly, pairwise correlations of AAWS are highest for healthy mice and decrease during the immune response (Figure 5). Therefore, we hypothesize that the average AAWS for healthy mice, shown in Figure 8 , are a signature of health. AAWS of infected mice, in turn, are systematically different from AAWS of healthy mice and can be separated by principal component analysis.

\section{Simulated unbiased antibody mixtures show ensemble properties}

In order to interpret the reported experimental results, we built a mathematical model based on the law of mass action. We defined a property vector $\vec{h}$ that characterizes each peptide's amino acid binding strength. In this model, the binding signals for a given simulated monoclonal antibody depend on the amino acid's position in a given peptide.

For a single simulated antibody, AAWS calculated by the amino acid composition-based linear regression model generally yield neither good predictive performance nor a high correlation with assigned AAWS $\vec{h}$. However, highly diverse antibody mixtures with random-in the sense of an independent identically distributed-repertoire, and no dominant antibodies, show both a close to perfect predictive performance and recovery of assigned AAWS $\vec{h}$ (Figure 4 and Additional file 5, Figure S5). Our mathematical model thus predicts that high predictive performance and high correlation of estimated AAWS and $\vec{h}$ are ensemble properties of such antibody mixtures: the average affinity of these mixtures does not depend on the epitope's amino acid position 
anymore. In contrast to that, the monoclonal antibodyepitope affinities do. We call random and highly diverse antibody mixtures that are not biased by dominant antibodies "unbiased". In fact, introducing, in simulations, a dominant antibody by increasing the concentration of a single antibody decreases predictive performance (Figure $6 \mathrm{~A})$. In addition, we showed that noise reduces predictive performance (Figure 6A).

\section{Serum samples of healthy BALB/c mice show signs of unbiased antibody mixtures}

As shown in our mathematical model, unbiased antibody mixtures are characterized by high predictive performance values. In view of the relatively high predictive performance of antibody binding profiles of serum samples from healthy BALB/c mice, we postulate that these sera exhibit properties of unbiased antibody mixtures.

The first prerequisite for an unbiased mixture is high diversity. This requirement seems to be met. The potential antibody diversity is very high [31], and the functional diversity is estimated to be of the order of $10^{4}$ [32]. However, fulfillment of the second requirement, the independent identical distribution of antibody binding sites, is harder to claim. On the one hand, the antibody repertoire is composed of preexisting gene segments and shaped by clonal selection, but on the other hand, V(D)J recombination and-in later stages of an immune response-somatic hypermutation arrange and mutate these segments in a largely random fashion [1]. Our results suggest that randomness in fact prevails. This is consistent with the hypothesis that antibody repertoires can potentially recognize the entire antigenic universe [33,34].

The predictive performance of healthy $\mathrm{BALB} / \mathrm{c}$ mice is not perfect but amounts to a median of 0.43 . This can be due to both noise and the fact that serum violates the assumptions of randomness to a certain degree. Noise may be caused by varying peptide spot quality on microarrays and by the experimental procedure itself. It is known that during a primary acute immune response, antibodies of a certain specificity for the antigen are produced in high abundance $[35,36]$. Therefore, it can be expected that sera of infected mice deviate from the properties of an unbiased mixture and would have reduced predictive performance values. Indeed, this is corroborated by experimental results (Figures 5 and 7).

Unbiased mixtures represent a special case for which the use of propensity scales for epitope prediction is justified The prediction of linear B-cell epitopes was first done by using propensity scales $[19,37,38]$. These scales assign a propensity value to every amino acid based on a priori studies of their physico-chemical properties. We found that our average AAWS, a posteriori termed signature of health (Figure 8 ), are not significantly correlated to widely used propensity scales.

Blythe and Flower tested 484 amino acid propensity scales on a set of 50 epitope-mapped proteins. They found that even the best set of scales perform only marginally better than random [39]. We show that unbiased mixtures represent a special case for which the converse holds true: antibody binding profiles of unbiased mixtures can be predicted based on AAWS. We show that the use of amino acid scales becomes increasingly less justified with increasing dominance of antibodies in a serum. In fact, each of Blythe and Flower's experiments used polyclonal antibodies raised against the whole protein [39]. We conjecture that the used polyclonal antibody mixtures were biased, that is, they contained dominant antibodies. In this regard, our study provides a possible explanation to Blythe and Flower's survey. More generally, our work suggests that results obtained with polyclonal antibody mixtures tend to be skewed by the inherent ensemble properties, which obscure the affinities of epitope-specific antibodies.

\section{Technological features may bias amino acid-associated weights}

We have shown that antibody mixtures exhibit ensemble properties. Resulting AAWS were shown to be consistent across healthy mice and qualitatively different from AAWS of infected mice (Figure 7). We have also provided a possible explanation for the difference between AAWS of healthy and infected mice: dominant antibodies in the course of the immune response.

However, the actual signature of health values shown in Figure 8 should be interpreted with caution. In addition to being indicative of both amino acid antibody binding preferences and physico-chemical properties (Figure 8 and Additional file 6, Figure S6), signal intensity may also be influenced by two other factors: (i) the accessibility of peptides and (ii) a possible interaction of aromatic amino acids and aromatic labeling dyes.

Accessibility may bias the resulting signal intensities systematically. For example, we find that cysteine contributes negatively to the signal intensity. This could partly be due to its ability to form disulfide bonds, which causes increased aggregation of cystein-containing peptides, and diminishes their surface exposure. This would lead to reduced antibody-peptide binding and accordingly to reduced signal intensity. Furthermore, it cannot be ruled out that aromatic amino acids interact via $\pi$ stacking with the aromatic labeling dyes Alexa Fluor 546 and 647 which are coupled to the secondary antibodies. Indeed, it has recently been found that TAMRA, another aromatic dye, cross-reacts with individual 
amino acids in a peptide sequence [40]. In order to minimize this effect, we performed secondary antibody correction on the log-transformed signal intensities.

\section{Conclusions}

We show that due to ensemble properties of unbiased mixtures, the position of amino acids in a linear epitope is no longer determinative for binding prediction. We found that prediction of peptide-binding as well as consistence of AAWS was best in sera of healthy BALB/c mice. Therefore, we defined a signature of health characterizing the binding behavior of serum of healthy individuals. This finding has far-reaching significance for the field of serological diagnostics.

Furthermore, our findings have also deep implications for the field of B cell epitope mapping as we have discovered an important special case which enables amino acid scale prediction of peptide binding. We show that amino acid scale prediction of peptide binding is justified only for unbiased mixtures. For other cases, alternative methods have to be sought. We thus showed that a knowledge of the composition of the used polyclonal mixture is essential for both the choice of the prediction method as well as the interpretation of results.

In the future, it will be of great interest to investigate the effects of a more detailed representation of binding in the mathematical model, and to study the effect of non-uniform antibody concentration distributions on predictive performance. Indeed, it has recently been shown for healthy zebrafish that the B cell clone repertoire follows a power-law distribution [41]. Thanks to our minimal assumptions approach, the conclusions of our model are independent of species, genetical background and individual exposure history. Future studies have to verify these predictions.

\section{Methods}

\section{Ethics Statement}

Animals were housed and handled following national guidelines and as approved by our animal ethics committee.

\section{Mice}

$\mathrm{BALB} / \mathrm{c}$ mice were bred and maintained under specific pathogen-free (SPF) conditions by the Department of Molecular Parasitology, Humboldt University Berlin, Berlin, Germany. Infection of mice with HB was carried out by oral gavage with 200 L3 stage larvae in distilled water.

\section{Sera}

Mice were narcotized and bled either by cardiac or retro-orbital puncture at the age of 8 weeks. Blood samples were collected from healthy SPF-BALB/c mice $(n=$
15), which were then infected with HB. Blood was collected at three time points post infection (dpi): at $10 \mathrm{dpi}$ $(n=15), 14 \mathrm{dpi}(n=13)$ and $18 \mathrm{dpi}(n=15)$. The blood was allowed to clot at room temperature and centrifuged. The supernatant was stored at $-20^{\circ} \mathrm{C}$.

\section{Monoclonal antibodies}

The 13 human monoclonal antibodies were kindly provided by the group of Hedda Wardemann (Max Planck Institute for Infection Biology, Berlin, Germany). Ten different Ig gene sequences of $\mathrm{IgG}^{+}$memory $\mathrm{B}$ cells from 2 healthy human donors, PN and VB, (PN115, PN138, PN16, PN89, VB1, VB142, VB161, VB176, VB18, VB4) [42] and three further ones from 3 other human donors ED38 [43], eiJB40 and mGO53 [44] were expressed as detailed in [45].

\section{Random peptide library}

The peptide library consists of 255 different 14-mer peptides. Their sequence was designed with a random generator. Repetitions of three or more consecutive amino acids were not allowed.

\section{Peptide synthesis and microarray design}

The peptide library was displayed in five identical subarrays on each slide purchased from JPT Peptide Technologies GmbH, Berlin, Germany. Furthermore, TAMRA-derived peptides, as internal fluorescence control, and mouse-IgM, mouse-IgG, human-IgM and human-IgG as secondary antibody controls, were included on each sub-array. Peptide microarrays were stored at $4^{\circ} \mathrm{C}$.

\section{Antibody binding assays}

The microarrays were briefly immersed in $100 \% \mathrm{v} / \mathrm{v}$ ethanol, washed three times with T-PBS (phosphate buffered saline containing $0.05 \% \mathrm{w} / \mathrm{v}$ Tween 20 ), three times with deionized water and dried by centrifugation. Since the microarray surfaces had been pre-treated to minimize unspecific binding of the target antibodies, no blocking step was required prior to incubation. All incubations were performed using a five-well adhesive incubation chamber (Multiwell GeneFrameTM, ABgene Germany, Hamburg, Germany) with a total assay volume of $45 \mu \mathrm{L}$ per well. Serum was diluted 1:10 in T-PBS and monoclonal antibodies were applied in a concentration of $10 \mu \mathrm{g} /$ $\mathrm{mL}$. We showed in a technological case study that approximately $10 \mu \mathrm{g} / \mathrm{ml}$ of antibody are best for reliable signal intensity measurements [14]. The concentration of IgM in in the serum of healthy SPF BALB/c mice was found to be around $0.50 \mathrm{mg} / \mathrm{ml}$ [46], which yields $50 \mu \mathrm{g} /$ $\mathrm{ml}$ for a 1:10 dilution. The diluted sera are thus within the optimal binding range. After incubation for four hours at room temperature, the microarrays were washed 
three times with T-PBS and three times with deionized water. Serum-antibody binding was detected with polyclonal goat anti-mouse IgM-Alexa Fluor 546 and polyclonal goat anti-mouse IgG-Alexa Fluor 647 (Invitrogen Ltd, Paisley, UK), simultaneously.

Monoclonal antibody binding was detected with polyclonal goat anti-human IgG Alexa Fluor 647 (Invitrogen Ltd, Paisley, UK). Secondary antibodies were diluted in T-PBS $(20 \mu \mathrm{g} / \mathrm{mL}, 300 \mu \mathrm{L})$ and incubated for one hour at room temperature. The microarrays were washed three times with T-PBS, three times with deionized water, rinsed with running deionized water and dried by centrifugation. Water, ethanol and PBS were filtered.

\section{Signal detection}

Fluorescence signals were measured on a GenePix microarray scanner (Molecular Devices GmbH, Ismaning, Germany) with a $532 \mathrm{~nm}$ laser using green $(\sim 550-600 \mathrm{~nm})$ emission filters and with $635 \mathrm{~nm}$ laser using red $(\sim 650$ $690 \mathrm{~nm}$ ) emission filters. An image file was generated at a resolution of $10 \mu \mathrm{m}$ using the scanner-associated GenePix ${ }^{\circledR}$ Pro software. Signal intensities were quantified with Genespotter ${ }^{\mathrm{TM}}$ software (MicroDiscovery GmbH, Berlin, Germany). Genespotter provides a fully automated gridfinding function, resulting in a reproducible read-out procedure. Signal intensities for each spot were calculated from a circular region around the center of the spot. Spots were examined for auto-fluorescence, but no relevant correlation between peptide composition and the fluorescence of clean microarrays was observed. Measured raw signal intensities were logtransformed $(\log (\mathrm{I}))$. Subsequently, the signal arising from the polyclonal secondary antibody was removed according to the linear model:

$$
\log (I)=\beta_{0}+\beta_{1} \log \left(I_{\text {SecondaryAntibody }}\right)+\varepsilon .
$$

By PLS-based computation of the intercepts, $\beta_{0}$ and $\beta_{1}$, we replaced $\log (I)$ with the resulting PLS-computed, mean-centered and scaled-to-unit variance residuals $\varepsilon$ for further analysis. The results reported in the main text of this paper are based exclusively on the calculated normalized residuals.

\section{Statistics}

The two-sided, non-paired Wilcoxon rank sum test was used to compute all p-values. P-values were regarded as significant when $p<0.05$. Association between variables was assessed by Pearson correlation $(r)$ unless otherwise stated.

\section{Generation of simulated signal intensities with a mathematical model}

Peptides and antibody binding sites were modeled as strings. Binding strengths between antibodies and the various amino acid residues of a peptide, referred to as assigned AAWS $\vec{h}$, were sampled from the uniform distribution on the closed interval 0[1]. A binding site on an antibody $\overrightarrow{a^{k}}$ was simulated in a similar fashion with a random number from the closed interval $[-1,1]$ for every sequential position and scaled such that $\left.\overrightarrow{\left(a^{k}\right.}\right)^{\mathrm{T}} \overrightarrow{a^{k}}=1$. The binding association between peptide $\overrightarrow{p^{i}}$ and antibody $\overrightarrow{a^{k}}$ was calculated by $y_{i, k}=\left(\overrightarrow{a^{k}}\right)^{\mathrm{T}} \overrightarrow{p^{i}}$

Based on the interpretation of the binding association as being negatively linearly proportional to the standard Gibbs free energy change of reaction, $\Delta_{r} G^{\circ}$, the binding affinity $K_{i, k}$, that is, the thermodynamic equilibrium association constant for antibody $k$ binding peptide $i$, is defined as shown in Equation 4.

$$
K_{i, k}=\exp \left(-\frac{\Delta_{r} G^{\mathrm{o}}}{R T}\right)=\exp \left(\frac{\beta_{0}+\beta_{1} \gamma_{i, k}}{R T}\right)
$$

Similar to a bit string model approach in [47], our approach to calculating $K_{i, k}$ assumes additivity in free energy of binding, an assumption that is supported by experimental results $[48,49]$. The signal intensity that we measure on the array is assumed to be proportional to the ratio of bound-to-total surface of the peptide spot, $S_{i}$. An expression for this quantity, based on the law of mass action, can be obtained from classical Langmuir adsorption theory [27] resulting in Equation 2 with $R=$ 8.314472, $T=273.15+25, \beta_{0}=0$ and $\beta_{1}=R T$.

At last, signal intensities were log-transformed, meancentered, and scaled to unit variance. If Gaussian noise $(\mathcal{N}(\mu=0, \sigma=0.01))$ was introduced into simulated signal intensities, the noise term was introduced before logarithmic transformation of the data. We showed that, for monoclonal antibodies, visibly fluorescent spots have at least a $K$-value of $10^{7} \mathrm{M}^{-1}[14]$.

\section{Partial least squares regression}

All calculations involving PLS were carried out with the pls package [50] for the $\mathrm{R}$ statistical programming environment [51].

\section{Model diagnostics}

The predictive performance is defined as:

$$
Q^{2}=1-\frac{\sum\left(\hat{s}_{\text {Leftout }}-s_{\text {Leftout }}\right)^{2}}{\sum s_{\text {Leftout }}^{2}} .
$$

The vector $\vec{s}_{\text {Leftout }}$ is the left-out test data set, the signal intensity of which is predicted ( $\hat{\vec{s}}_{\text {Leftout }}$ ) from the remaining training data set. The left-out test data represented randomly chosen $10 \%$ of the total data set. 


\section{Principal component analysis}

Principal component analysis was performed using the pcaMethods R-package [52].

\section{Additional material}

\begin{abstract}
Additional File 1: Supporting Figure S1: Experimental setup: infection of BALB/c mice with Heligmosomoides bakeri and collection of blood samples at three different stages of immune response. Serum samples from $15 \mathrm{BALB} / \mathrm{C}$ mice raised under specific pathogen-free conditions were collected. These mice were infected with the intestinal nematode Heligmosomoides bakeri formerly known as Heligmosomoides polygyrus [53]. Further serum samples were collected at $10 \mathrm{dpi}$ (days post infection; 15 samples), at $14 \mathrm{dpi}$ (13 samples), and at 18 dpi (15 samples) totaling 58 serum samples. The serum was isolated and subsequently incubated with random peptide libraries. We categorized the serum samples into healthy (0 dpi; 15 samples), acute phase (10 and $14 \mathrm{dpi} ; 15$ and 13 samples respectively) and early chronic phase (18 dpi; 15 samples), thus delineating the three major stages of immune response of a mouse, before and after primary infection with HB. Practical experimental difficulties reduced the intended number of usable 14 dpi samples from 15 to 13 .
\end{abstract}

Additional File 2: Supporting Figure S2: Removing the signal of the secondary antibody accentuates differences between binding profiles of monoclonal and serum antibodies. (A) The predictive performance values $\left(Q^{2}\right)$ were calculated for monoclonal $(\mathrm{mAb})$ as well as serum $\operatorname{lgM}(\mathrm{s} \lg M)$ and $\lg \mathrm{G}(\mathrm{s} \lg \mathrm{G})$ antibody binding profiles before (blue) and after (red) correction of the measured log-transformed signal intensities by removal of the polyclonal secondary antibody-correlated signals using PLS. (B) Shown is the pairwise correlation ( $r$ ) of the corresponding AAWS $\vec{w}^{j}$. For the two statistical measures, signal correction entails a significant decrease in the mAb median, whereas slgM and slgG medians remain largely unchanged. Both before and after secondary antibody correction of antibody binding profiles, slgM profiles have higher predictive performance $\left(Q^{2}\right)$ and a higher median pairwise correlation ( $r$ ) of AAWS than slgG profiles. In (A) and (B), mAb signifies antibody binding profiles from 13 monoclonal antibodies and slgM/slgG serum IgM and serum IgG binding profiles from 58 BALB/C mice sera, respectively. Antibody binding profiles were measured with the standard peptide library of 25514 -mers. Corresponding AAWS $\left(\vec{w}^{j}\right)$ were determined with Equation 1.

Additional File 3: Supporting Figure S3: Predictive performance and pairwise correlation of amino acid-associated weights are higher for serum IgG than for monoclonal antibodies. (A) Predictive performance values $\left(Q^{2}\right)$ were calculated for monoclonal (mAb) and serum IgG antibody (Sera lgG) binding profiles. (B) Shown is the pairwise correlation (r) of the corresponding AAWS $\vec{w}^{j}$. In both (A) and (B) mAb signifies antibody binding profiles from 13 monoclonal antibodies and Sera IgG binding profiles from $58 \mathrm{BALB} / \mathrm{c}$ mice sera. Differences in predictive performance $\left(Q^{2}\right)$ and pairwise correlation ( $r$ ) of AAWS between monoclonal and serum IgG antibodies are significant $(p<$ 0.001). Antibody binding profiles were measured with the standard peptide library of 255 14-mers. Corresponding AAWS $\left(\vec{w}^{j}\right)$ were determined with Equation 1.

Additional File 4: Supporting Figure S4: Predictive performance and pairwise correlation of amino acid-associated weights decrease for serum IgG antibodies during the course of the immune response. (A) Predictive performance values $\left(Q^{2}\right)$ were computed from serum lgG antibody binding profiles across three stages of immune response: healthy, acute, early chronic. (B) Shown is the pairwise correlation (r) of the corresponding AAWS $\vec{w}_{\text {sim }}^{j}$. Number of BALB/C mice serum samples: 15 from healthy mice, after infection with $\mathrm{HB}$ : 15 samples taken at $10 \mathrm{dpi}$ and 13 samples taken at $14 \mathrm{dpi}$ (acute phase) and 15 samples taken at $18 \mathrm{dpi}$ (early chronic) totaling $58 \mathrm{BALB} / \mathrm{c}$ serum samples. Differences in predictive performance $\left(Q^{2}\right)$ between both healthy and early chronic phase mice and healthy and acute phase mice are significant $(p<0.05)$ as are differences in pairwise correlation $(r)$ between all three stages of immune response $(p<0.001)$. Antibody binding profiles were measured with the standard peptide library of 255 14-mers. Corresponding AAWS $\left(\vec{w}^{j}\right)$ were computed using Equation 1.

Additional File 5: Supporting Figure S5: Simulations show that recovery of assigned amino acid-associated weights is positively correlated to antibody diversity. This Figure is complementary to Figure 3. Antibody binding profiles were simulated for antibody mixtures of 1 to 16348 different antibodies. The correlation $(r)$ of simulated AAWS $\left(\left(\vec{w}_{\text {sim }}^{i}\right)\right)$ with assigned AAWS $(\vec{h})$ increases with increasing antibody diversity. Both a simulated random peptide library $\left(\mathbf{X}_{\text {sim }}\right)$ of 255 14-mers as well as assigned AAWS $\vec{h}$ were generated once and kept constant across the whole simulation. Simulated antibody binding profiles were computed with Equation 2, detailed in the results section. Corresponding AAWS were determined with Equation 1. For every mixture of $n_{\mathrm{Ab}}$ different antibodies, 100 simulations with newly randomly generated antibody mixtures were run.

Additional File 6: Supporting Figure S6: Correlation between 26 physico-chemical properties and the average AAWS of healthy mice. The average AAWS of healthy mice were correlated with the $z$ scale published by Sandberg and colleagues [29]. The shown correlation coefficients are Spearman-Rank-correlation coefficients. Same abbreviations were used as by Sandberg and colleagues [29]. MW (molecular weight), TLx (thin layer chromatography at various conditions), vdW (side chain van der Waals volume), NMx (NMR-proton shift at $\mathrm{pD}=\mathrm{x}), \log \mathrm{P}(10 \log$ (octanol/water) partition coefficient), EHOMO (energy of highest occupied molecular orbital), ELUMO (energy of lowest unoccupied molecular orbital), HOF (heat of formation), POLAR $(\alpha$-polarizability), EN (absolute electronegativity), HA (absolute hardness), Stot (total accessible molecular surface area), Spol (polar accessible molecular surface area), Snp (non-polar accessible molecular surface area), HDONR (number of hydrogen bond donors), HACCR (number of hydrogen bond acceptors), Chpos (indicator of positive charge in side chain), Chneg (indicator of negative charge in side chain). Legend: Red, positive correlation coefficients; blue, negative correlation coefficients.

\section{List of Abbreviations}

AACM: Amino acid composition matrix; AAWS: Amino acid-associated weights; FABR: Functional antibody repertoire; HB: Heligmosomoides bakeri; PLS: Partial least squares regression.

\section{Acknowledgements}

We thank René Riedel (German Rheumatism Research Center, Berlin, Germany), Carsten C. Mahrenholz (Charité - University Medicine Berlin, Berlin, Germany), Johannes Eckstein and Nicole Wittenbrink (Systems Immunology Lab, Dept. of Biology, Humboldt University Berlin, Berlin, Germany) for critical reading of the manuscript. We thank $\mathrm{H}$. Wardemann (Max Planck Institute for Infection Biology, Berlin, Germany) and her lab for providing the monoclonal antibodies. The authors were funded by BMBF Grant 315005B and the ProFit Grant IBB/EFRES (10142548).

\section{Author details}

'Systems Immunology Lab, Department of Biology, Humboldt University Berlin, and Research Center ImmunoSciences, Charité University Medicine Berlin, Berlin, Germany. ${ }^{2}$ Bayer Cropscience N.V., Technologiepark, 38, 9052 Zwijnaarde, Gent, Belgium. ${ }^{3}$ Studienmethodik und Statistik, Universitätsspital Basel, Basel, Switzerland. ${ }^{4}$ Department of Molecular Parasitology, Humboldt University Berlin, Berlin, Germany. ${ }^{5}$ MicroDiscovery GmbH, Berlin, Germany. ${ }^{6}$ Contributed equally to this study.

\section{Authors' contributions}

MOG conceived of the project. $H R$, VG and MOG designed the research. $H R$, JS, VG and MOG analyzed data. HR, VG and MOG wrote the manuscript. NB and $J$ performed peptide array experiments. SR and SH provided murine sera. All authors discussed results. All authors have read and approved the final manuscript. 
Received: 11 June 2011 Accepted: 21 February 2012

Published: 21 February 2012

\section{References}

1. Abbas AK, Lichtman A: Cellular and Molecular Immunology. 5 edition. Saunders; 2005.

2. Nobrega A, Grandien A, Haury M, Hecker L, Malanchère E, Coutinho A Functional diversity and clonal frequencies of reactivity in the available antibody repertoire. European Journal of Immunology 1998, 28(4):1204-1215.

3. Quintana FJJ, Merbl Y, Sahar E, Domany E, Cohen IR: Antigen-chip technology for accessing global information about the state of the body. Lupus 2006, 15(7):428-430.

4. Nobrega A, Haury M, Grandien A, Malanchère E, Sundblad A, Coutinho A: Global analysis of antibody repertoires. II. Evidence for specificity, selfselection and the immunological "homunculus" of antibodies in normal serum. European Journal of Immunology 1993, 23(11):2851-2859.

5. Haury M, Grandien A, Sundblad A, Coutinho A, Nobrega A: Global analysis of antibody repertoires. 1 . An immunoblot method for the quantitative screening of a large number of reactivities. Scandinavian Journal of Immunology 1994, 39:79-87.

6. Robinson WH, DiGennaro C, Hueber W, Haab BB, Kamachi M, Dean EJ, Fournel S, Fong D, Genovese MC, de Vegvar HEN, Skriner K, Hirschberg DL, Morris RI, Muller S, Pruijn GJ, van Venrooij WJ, Smolen JS, Brown PO, Steinman L, Utz PJ: Autoantigen microarrays for multiplex characterization of autoantibody responses. Nat Med 2002, 8(3):295-301.

7. Quintana FJ: Functional immunomics: Microarray analysis of lgG autoantibody repertoires predicts the future response of mice to induced diabetes. Proceedings of the National Academy of Sciences 2004 101(suppl 2):14615-14621.

8. Merbl Y, Itzchak R, Vider-Shalit T, Louzoun Y, Quintana FJ, Vadai E, Eisenbach L, Cohen IR: A systems immunology approach to the hosttumor interaction: large-scale patterns of natural autoantibodies distinguish healthy and tumor-bearing mice. PloS One 2009, 4(6):e6053.

9. Cekaite L, Haug O, Myklebost O, Aldrin M, Østenstad B, Holden M, Frigessi $A$, Hovig $E$, Sioud $M$ : Analysis of the humoral immune response to immunoselected phage-displayed peptides by a microarray-based method. PROTEOMICS 2004, 4(9):2572-2582.

10. Bongartz J, Bruni N, Or-Guil M: Epitope mapping using randomly generated peptide libraries. Methods in Molecular Biology (Clifton, N.J.) 2009, 524:237-246.

11. Legutki JB, Magee DM, Stafford P, Johnston SA: A general method for characterization of humoral immunity induced by a vaccine or infection. Vaccine 2010, 28(28):4529-4537.

12. Reddy MM, Wilson R, Wilson J, Connell S, Gocke A, Hynan L, German D, Kodadek T: Identification of Candidate IgG Biomarkers for Alzheimer's Disease via Combinatorial Library Screening. Cell 2011, 144:132-142.

13. Weiser AA, Or-Guil M, Tapia V, Leichsenring A, Schuchhardt J, Frömmel C, Volkmer-Engert R: SPOT synthesis: Reliability of array-based measurement of peptide binding affinity. Analytical Biochemistry 2005, 342(2):300-311.

14. Tapia V, Bongartz J, Schutkowski M, Bruni N, Weiser A, Ay B, Volkmer R, OrGuil M: Affinity profiling using the peptide microarray technology: A case study. Analytical Biochemistry 2007, 363:108-118.

15. Brown JR, Stafford P, Johnston SA, Dinu V: Statistical methods for analyzing immunosignatures. BMC Bioinformatics 2011, 12:349.

16. Chou PY, Fasman GD: Prediction of the secondary structure of proteins from their amino acid sequence. Advances in Enzymology and Related Areas of Molecular Biology 1978, 47:45-148.

17. Parker JM, Guo D, Hodges RS: New hydrophilicity scale derived from high-performance liquid chromatography peptide retention data: correlation of predicted surface residues with antigenicity and X-rayderived accessible sites. Biochemistry 1986, 25(19):5425-5432

18. Emini EA, Hughes JV, Perlow DS, Boger J: Induction of hepatitis A virusneutralizing antibody by a virus-specific synthetic peptide. Journal of Virology 1985, 55(3):836-839.

19. Hopp TP, Woods KR: Prediction of protein antigenic determinants from amino acid sequences. Proceedings of the National Academy of Sciences of the USA 1981, 78(6):3824-3828.

20. Mandrika I, Prusis P, Yahorava S, Tars K, Wikberg JE: QSAR of multiple mutated antibodies. Journal of Molecular Recognition 2007, 20(2):97-102.
21. Mandrika I, Prusis P, Yahorava S, Shikhagaie M, Wikberg JE: Proteochemometric modelling of antibody-antigen interactions using SPOT synthesised peptide arrays. Protein Engineering, Design and Selection 2007, gzm022

22. Mandrika I, Prusis P, Bergström J, Yahorava S, Wikberg JES: Improving the affinity of antigens for mutated antibodies by use of statistical molecular design. Journal of Peptide Science: An Official Publication of the European Peptide Society 2008, 14(7):786-796

23. De Boer RJ, Perelson AS: T Cell Repertoires and Competitive Exclusion Journal of Theoretical Biology 1994, 169(4):375-390.

24. Perelson AS: Immune Network Theory. Immunological Reviews 1989, 110:5-36.

25. Sulzer B, Perelson AS: Equilibrium binding of multivalent ligands to cells: Effects of cell and receptor density. Mathematical Biosciences 1996, 135(2):147-185.

26. Perelson AS, Weisbuch G: Immunology for physicists. Reviews of Modern Physics 1997, 69(4):1219.

27. Alkhamis KA, Wurster DE: Prediction of adsorption from multicomponent solutions by activated carbon using single-solute parameters. Part IIProposed equation. AAPS PharmSciTech 2002, 3(3):E23.

28. Rausch S, Huehn J, Kirchhoff D, Rzepecka J, Schnoeller C, Pillai S, Loddenkemper C, Scheffold A, Hamann A, Lucius R, Hartmann S: Functional Analysis of Effector and Regulatory T Cells in a Parasitic Nematode Infection. Infect Immun 2008, 76(5):1908-1919.

29. Sandberg M, Eriksson L, Jonsson J, Sjöström M, Wold S: New chemical descriptors relevant for the design of biologically active peptides. $A$ multivariate characterization of 87 amino acids. Journal of Medicinal Chemistry 1998, 41(14):2481-2491.

30. Kolaskar A, Tongaonkar PC: A semi-empirical method for prediction of antigenic determinants on protein antigens. FEBS Letters 1990, 276(12):172-174.

31. Berek C, Griffiths GM, Milstein C: Molecular events during maturation of the immune response to oxazolone. Nature 1985, 316(6027):412-418.

32. Brissac C, Nobrega A, Carneiro J, Stewart J: Functional diversity of natural IgM. Int Immunol 1999, 11(9):1501-1507.

33. Jerne NK: The natural-selection theory of antibody formation. Proceedings of the National Academy of Sciences of the USA 1955, 41(11):849.

34. Perelson AS, Oster GF: Theoretical studies of clonal selection: Minimal antibody repertoire size and reliability of self-non-self discrimination. Journal of Theoretical Biology 1979, 81(4):645-670.

35. Janeway C, Shlomchik MJ, Walport : Immunobiology. 6 edition. Garland Science; 2004

36. McCoy KD, Stoel M, Stettler R, Merky P, Fink K, Senn BM, Schaer C, Massacand J, Odermatt B, Oettgen HC, Zinkernagel RM, Bos NA, Hengartner H, Macpherson AJ, Harris NL: Polyclonal and Specific Antibodies Mediate Protective Immunity against Enteric Helminth Infection. Cell Host \& Microbe 2008, 4(4):362-373.

37. Greenbaum JA, Andersen PH, Blythe M, Bui H, Cachau RE, Crowe J, Davies M, Kolaskar AS, Lund O, Morrison S, Mumey B, Ofran Y, Pellequer J, Pinilla C, Ponomarenko JV, Raghava GPS, van Regenmortel MHV, Roggen EL, Sette A, Schlessinger A, Sollner J, Zand M, Peters B: Towards a consensus on datasets and evaluation metrics for developing B-cell epitope prediction tools. Journal of Molecular Recognition 2007, 20(2):75-82.

38. EL-Manzalawy $Y$, Honavar $V$ : Recent advances in B-cell epitope prediction methods. Immunome Research 2010, 6(Suppl 2):S2.

39. Blythe MJ, Flower DR: Benchmarking B cell epitope prediction: Underperformance of existing methods. Protein Science: A Publication of the Protein Society 2005, 14:246-248.

40. Mahrenholz CC, Tapia V, Stigler RD, Volkmer R: A study to assess the crossreactivity of cellulose membrane-bound peptides with detection systems: an analysis at the amino acid level. Journal of Peptide Science: An Official Publication of the European Peptide Society 2010, 16(6):297-302.

41. Weinstein JA, Jiang N, White RA, Fisher DS, Quake SR: High-Throughput Sequencing of the Zebrafish Antibody Repertoire. Science 2009, 324(5928):807-810

42. Tiller T, Tsuiji M, Yurasov S, Velinzon K, Nussenzweig MC, Wardemann H: Autoreactivity in human IgG+ memory B cells. Immunity 2007, 26(2):205-213

43. Meffre E, Schaefer A, Wardemann H, Wilson P, Davis E, Nussenzweig MC Surrogate Light Chain Expressing Human Peripheral B Cells Produce 
Self-reactive Antibodies. The Journal of Experimental Medicine 2004, 199:145-150.

44. Wardemann H, Yurasov S, Schaefer A, Young JW, Meffre E,

Nussenzweig MC: Predominant autoantibody production by early human

B cell precursors. Science (New York, N.Y.) 2003, 301(5638):1374-1377.

45. Tiller T, Meffre E, Yurasov S, Tsuiji M, Nussenzweig MC, Wardemann H:

Efficient generation of monoclonal antibodies from single human B cells by single cell RT-PCR and expression vector cloning. Journal of immunological methods 2008, 329(1-2):112-124.

46. Haury M, Sundblad A, Grandien A, Barreau C, Coutinho A, Nobrega A: The repertoire of serum IgM in normal mice is largely independent of external antigenic contact. European journal of immunology 1997, 27(6):1557-1563.

47. Rosenwald S, Kafri R, Lancet D: Test of a Statistical Model for Molecular Recognition in Biological Repertoires. Journal of Theoretical Biology 2002, 216(3):327-336.

48. Horovitz A, Rigbi M: Protein-protein interactions: Additivity of the free energies of association of amino acid residues. Journal of Theoretical Biology 1985, 116:149-159.

49. Free SM, Wilson JW: A Mathematical Contribution to Structure-Activity Studies. Journal of Medicinal Chemistry 1964, 7(4):395-399.

50. Mevik B, Wehrens R: The pls Package: Principal Component and Partial Least Squares Regression in R. Journal of Statistical Software 2007, 18(2): $1-24$

51. Team RDC: R: A Language and Environment for Statistical Computing Vienna, Austria; 2009.

52. Stacklies W, Redestig H, Scholz M, Walther D, Selbig J: pcaMethods a bioconductor package providing PCA methods for incomplete data. Bioinformatics 2007, 23(9):1164-1167.

53. Behnke J, Harris PD: Heligmosomoides bakeri: a new name for an old worm? Trends in Parasitology 2010.

doi:10.1186/1471-2164-13-79

Cite this article as: Greiff et al: A minimal model of peptide binding predicts ensemble properties of serum antibodies. BMC Genomics 2012 13:79.

\section{Submit your next manuscript to BioMed Central and take full advantage of:}

- Convenient online submission

- Thorough peer review

- No space constraints or color figure charges

- Immediate publication on acceptance

- Inclusion in PubMed, CAS, Scopus and Google Scholar

- Research which is freely available for redistribution

Submit your manuscript at www.biomedcentral.com/submit 